\title{
LOW ENERGY SOLUTIONS FOR SINGULARLY PERTURBED COUPLED NONLINEAR SYSTEMS ON A RIEMANNIAN MANIFOLD WITH BOUNDARY.
}

\author{
MARCO GHIMENTI AND ANNA MARIA MICHELETTI
}

\begin{abstract}
Let $(M, g)$ be a smooth, compact Riemannian manifold with smooth boundary, with $n=\operatorname{dim} M=2,3$. We suppose the boundary $\partial M$ to be a smooth submanifold of $M$ with dimension $n-1$. We consider a singularly perturbed nonlinear system, namely Klein-Gordon-Maxwell-Proca system, or Klein-Gordon-Maxwell system of Scrhoedinger-Maxwell system on $M$. We prove that the number of low energy solutions, when the perturbation parameter is small, depends on the topological properties of the boundary $\partial M$, by means of the Lusternik Schnirelmann category. Also, these solutions have a unique maximum point that lies on the boundary.
\end{abstract}

\section{INTRODUCTION}

Let $(M, g)$ be a smooth, compact Riemannian manifold with smooth boundary, with $n=\operatorname{dim} M=2,3$. We suppose the boundary $\partial M$ to be a smooth submanifold of $M$ with dimension $n-1$.

We consider the following singularly perturbed electrostatic Klein-Gordon-MaxwellProca (shortly KGMP) system on $M$ with Neumann boundary condition

$$
\left\{\begin{array}{cc}
-\varepsilon^{2} \Delta_{g} u+a u=|u|^{p-2} u+\omega^{2}(q v-1)^{2} u & \text { in } M \\
-\Delta_{g} v+\left(1+q^{2} u^{2}\right) v=q u^{2} & \text { in } M \\
\frac{\partial u}{\partial \nu}=0, \frac{\partial v}{\partial \nu}=0 & \text { on } \partial M
\end{array}\right.
$$

Here $\varepsilon>0, a>0, q>0, \omega \in(-\sqrt{a}, \sqrt{a})$ and $4 \leq p<2^{*}$ being $2^{*}=6$ for $n=3$ or $2^{*}=+\infty$ for $n=2$.

The Neumann condition for the function $u$ is interesting since it shows how the topological properties of the boundary influence the number of solutions of (11). Moreover from a physical viewpoint, give a Neumann condition for the second function $v$ corresponds to fix the electrical field on $\partial M$ which is a natural condition (for a more detailed discussion on this topic, we refer to [8, 10]).

The study of KGMP systems recently has known a rise of interest in the mathematical community. In [13, 14, 15] equation (1) has been studied on a Riemaniann boundariless manifold $M$. A similar problem has been considered in a flat domain

2010 Mathematics Subject Classification. 35J60, 35J20,53C80,81V10.

Key words and phrases. Riemannian manifolds with boundary, Klein-Gordon-Maxwell systems, Scrhoedinger-Maxwell systems, Lusternik Schnirelmann category, one peak solutions.

The authors were partially supported by 2014 GNAMPA project: "Equazioni di campo nonlineari: solitoni e dispersione". 
$\Omega$ by D'Aprile and Wei $[5,6$. In the context of flat domains, moreover, many authors have dealt with Klein Gordon Maxwell systems without singular perturbation in the Laplacian term [1, 2, 3, 7, 9, 16.

In this paper we prove the following result.

Theorem 1. For $\varepsilon$ small enough the KGMP system (1) has at least cat $\partial M$ non constant distinct solutions $\left(u_{\varepsilon}, v_{\varepsilon}\right)$ with low energy. Here cat $\partial M$ is the Lusternik Schnirelmann category. Moreover the functions $u_{\varepsilon}$ have a unique maximum point $P_{\varepsilon} \in \partial M$ and $u_{\varepsilon}=Z_{\varepsilon, P_{\varepsilon}}+\Psi_{\varepsilon}$ where $Z_{\varepsilon, P_{\varepsilon}}$ is defined in (6) and $\left\|\Psi_{\varepsilon}\right\|_{L^{\infty}(M)} \rightarrow 0$.

Remark 2. We notice that the same result can be obtained verbatim for the electrostatic Klein-Gordon-Maxwell (shortly KGM) system with Neumann/Dirichlet boundary condition,

$$
\left\{\begin{array}{cc}
-\varepsilon^{2} \Delta_{g} u+a u=|u|^{p-2} u+\omega^{2}(q v-1)^{2} u & \text { in } M \\
-\Delta_{g} v+q^{2} u^{2} v=q u^{2} & \text { in } M \\
\frac{\partial u}{\partial \nu}=0, v=0 & \text { on } \partial M
\end{array}\right.
$$

and for the Schroedinger-Maxwell system with Neumann/Dirichlet boundary condition, for $\varepsilon>0, a>0, q>0, \omega \in \mathbb{R}$ and $4<p<2^{*}$

$$
\left\{\begin{array}{cc}
-\varepsilon^{2} \Delta_{g} u+a u+\omega u v=|u|^{p-2} u & \text { in } M \\
-\Delta_{g} v=q u^{2} & \text { in } M \\
\frac{\partial u}{\partial \nu}=0, v=0 & \text { on } \partial M
\end{array}\right.
$$

We explicitly treat systems (11) and (2) in the paper, pointing out the differences in the proofs whenever necessary. For system (3) the estimates are easier and left to the reader. We just mention that we have to rule out the case $p=4$ in order to have a smooth Nehari manifold (cfr. section 3)

Remark 3 . The result of this paper relies on the topology of the boundary $\partial M$. In a forthcoming paper the authors will point out how the geometry of $\partial M$ affects the number of one peaked solutions.

The paper is structured as follows: in Section 2 some basic concepts are recalled and it is introduced the variational structure of the problem. The Nehari manifold that is a natural constraint for the variational problem is introduced in Section 3. Section 4 contains the lines of the proof of Theorem 11 while in sections 5 , 6 and 7 the steps of the proof are explained in full details. The profile description is contained in Section 8. Some technical result is postponed in Section 9 to do not overload the presentation of the results.

\section{Preliminaries}

We recall some well know result on Riemaniann manifold with boundary. At first we introduce a coordinates system for a neighborhood of the boundary $\partial M$.

If $\xi$ belongs to $\partial M$, let $\bar{y}=\left(y_{1}, \ldots, y_{n-1}\right)$ be Riemannian normal coordinates on the $n-1$ manifold $\partial M$ at the point $\xi$. For a point $x \in M$ close to $\xi$, there exists a unique $\bar{x} \in \partial M$ such that $d_{g}(x, \partial \mathcal{M})=d_{g}(x, \bar{x})$. We set $\bar{y}(x) \in \mathbb{R}^{n-1}$ the normal coordinates for $\bar{x}$ and $y_{n}(x)=d_{g}(x, \partial \mathcal{M})$. Then we define a chart $\Psi_{\xi}^{\partial}: \mathbb{R}_{+}^{n} \rightarrow M$ such that $\left(\bar{y}(x), y_{n}(x)\right)=\left(\Psi_{\xi}^{\partial}\right)^{-1}(x)$. These coordinates are called Fermi coordinates at $\xi \in \partial M$.

We note by $d_{g}^{\partial}$ and $\exp ^{\partial}$ respectively the geodesic distance and the exponential map on by $\partial M$. 
We define the following neighborhood of a point $\xi \in \partial M$

$$
I_{\xi}(\rho, R)=\left\{x \in M: y_{n}=d_{g}(x, \partial M)<\rho \text { and }|\bar{y}|=d_{g}^{\partial}\left(\exp _{q}^{\partial}(\bar{y}(x)), \xi\right)<R\right\} \text {. }
$$

where $R, \rho>0$ are smaller than the injectivity radius of $M$. Often we will denote $I_{\xi}(R)=I_{\xi}(R, R)$ and, if no ambiguity is present, we simply use $I_{\xi}$ for $I_{\xi}(R, \rho)$ or for $I_{\xi}(R)$.

Let $\mathbb{R}_{+}^{n}=\left\{y=\left(\bar{y}, y_{n}\right): \bar{y} \in \mathbb{R}^{n-1}, y_{n} \geq 0\right\}$. It is well known that there exists a least energy solution $V \in H^{1}\left(\mathbb{R}_{+}^{n}\right)$ of the equation

$$
\left\{\begin{array}{c}
-\Delta V+\left(a-\omega^{2}\right) V=|V|^{p-2} V, V>0 \quad \text { on } \mathbb{R}_{+}^{n} \\
\left.\frac{\partial V}{\partial y_{n}}\right|_{(\bar{y}, 0)}=0 .
\end{array}\right.
$$

We remark that, set $U$ the least energy solution of

$$
\left\{\begin{array}{c}
-\Delta U+\left(a-\omega^{2}\right) U=|U|^{p-2} U, U>0 \quad \text { on } \mathbb{R}^{n} \\
U \in H^{1}\left(\mathbb{R}^{n}\right)
\end{array}\right.
$$

which is radially symmetric, we have that $V=\left.U\right|_{y_{n} \geq 0}$.

Set $V_{\varepsilon}(y)=V\left(\frac{y}{\varepsilon}\right)$, and fixed $\xi \in \partial M$ we define the function $Z_{\varepsilon, \xi}(x)$ as

$$
Z_{\varepsilon, \xi}(x)=\left\{\begin{array}{cc}
V_{\varepsilon}(y(x)) \chi_{R}(|\bar{y}(x)|) \chi_{\rho}\left(y_{n}(x)\right) & \text { if } x \in I_{\xi} \\
0 & \text { otherwise }
\end{array}\right.
$$

where $\chi_{T}: \mathbb{R}^{+}: \rightarrow[0,1]$ is a smooth cut off function such that $\chi_{T}(s) \equiv 1$ for $0 \leq s \leq T / 2, \chi_{R}(s) \equiv 0$ for $s \geq T$ and $\left|\tilde{\chi}_{T}^{\prime}(s)\right| \leq 1 / T$.

We endow $H_{g}^{1}(M)$ with the scalar product and norm

$$
\langle u, v\rangle_{\varepsilon}:=\frac{1}{\varepsilon^{n}} \int_{M} \varepsilon^{2} \nabla_{g} u \nabla_{g} v+\left(a-\omega^{2}\right) u v d \mu_{g} ; \quad\|u\|_{\varepsilon}=\langle u, u\rangle_{\varepsilon}^{1 / 2} .
$$

We call $H_{\varepsilon}$ the space $H_{g}^{1}$ equipped with the norm $\|\cdot\|_{\varepsilon}$. We also define $L_{\varepsilon}^{p}$ as the space $L_{g}^{p}(M)$ endowed with the norm $|u|_{\varepsilon, p}=\frac{1}{\varepsilon^{n}}\left(\int_{M} u^{p} d \mu_{g}\right)^{1 / p}$. We also use the obvious notation $H_{0, \varepsilon}$ for the space $H_{0, g}^{1}$ with the norm $\|\cdot\|_{\varepsilon}$, where $H_{g}^{1}$ (resp. $H_{0, g}^{1}$ ) is the closure of $C^{\infty}(M)$ (resp. $C_{0}^{\infty}(M)$ ) with respect to the norm $\int_{M}\left|\nabla_{g} u\right|^{2}+u^{2}$ (resp. $\int_{M}\left|\nabla_{g} u\right|^{2}$ ).

2.1. The function $\psi$. First of all, we reduce the system to a single equation. In order to overcome the problems given by the competition between $u$ and $v$, using an idea of Benci and Fortunato [2, we introduce the map $\psi$ defined by the equation

$$
\left\{\begin{array}{cc}
-\Delta_{g} \psi+\left(1+q^{2} u^{2}\right) \psi=q u^{2} & \text { in } M \\
\frac{\partial \psi}{\partial \nu}=0 & \text { on } \partial M
\end{array}\right.
$$

in case of Neumann boundary condition or by

$$
\left\{\begin{array}{cc}
-\Delta_{g} \psi+q u^{2} \psi=q u^{2} & \text { in } M \\
\psi=0 & \text { on } \partial M
\end{array}\right.
$$

in case of Dirichlet boundary condition.

In what follows we call $H=H_{g}^{1}$ for the Neumann problem and $H=H_{0, g}^{1}$ for the Dirichelt problem. Thus with abuse of language we will say that $\psi: H \rightarrow H$ in both (7) and (8). Moreover, from standard variational arguments, it easy to see that $\psi$ is well-defined in $H$ and it holds

$$
0 \leq \psi(u) \leq 1 / q
$$


for all $u \in H$.

Lemma 4. The map $\psi: H \rightarrow H$ is $C^{2}$ and its differential $\psi^{\prime}(u)[h]=V_{u}[h]$ at $u$ is the map defined by

$$
-\Delta_{g} V_{u}[h]+\left(1+q^{2} u^{2}\right) V_{u}[h]=2 q u(1-q \psi(u)) h \text { for all } h \in H .
$$

in case of Neumann boundary condition or

$$
-\Delta_{g} V_{u}[h]+q^{2} u^{2} V_{u}[h]=2 q u(1-q \psi(u)) h \text { for all } h \in H .
$$

in case of Dirichlet boundary condition.

Also, we have

$$
0 \leq \psi^{\prime}(u)[u] \leq \frac{2}{q} .
$$

Finally, the second derivative $(h, k) \rightarrow \psi^{\prime \prime}(u)[h, k]=T_{u}(h, k)$ is the map defined by the equation

$$
-\Delta_{g} T_{u}(h, k)+\left(1+q^{2} u^{2}\right) T_{u}(h, k)=-2 q^{2} u\left(k V_{u}(h)+h V_{u}(k)\right)+2 q(1-q \psi(u)) h k
$$

in case of Neumann boundary condition or

$$
-\Delta_{g} T_{u}(h, k)+q^{2} u^{2} T_{u}(h, k)=-2 q^{2} u\left(k V_{u}(h)+h V_{u}(k)\right)+2 q(1-q \psi(u)) h k
$$

in case of Dirichlet boundary condition.

Lemma 5. The map $\Theta: H \rightarrow \mathbb{R}$ given by

$$
\Theta(u)=\frac{1}{2} \int_{M}(1-q \psi(u)) u^{2} d \mu_{g}
$$

is $C^{2}$ and

for any $u, h \in H$

$$
\Theta^{\prime}(u)[h]=\int_{M}(1-q \psi(u))^{2} u h d \mu_{g}
$$

For the proofs of these results we refer to [11, in which the case of KGMP is treated. For KGM systems, the proof is identical.

Now, we introduce the functionals $I_{\varepsilon}, J_{\varepsilon}, G_{\varepsilon}: H \rightarrow \mathbb{R}$

$$
I_{\varepsilon}(u)=J_{\varepsilon}(u)+\frac{\omega^{2}}{2} G_{\varepsilon}(u),
$$

where

$$
J_{\varepsilon}(u):=\frac{1}{2 \varepsilon^{n}} \int_{M}\left[\varepsilon^{2}\left|\nabla_{g} u\right|^{2}+\left(a-\omega^{2}\right) u^{2}\right] d \mu_{g}-\frac{1}{p \varepsilon^{n}} \int_{M}\left(u^{+}\right)^{p} d \mu_{g}
$$

and

$$
G_{\varepsilon}(u):=\frac{1}{\varepsilon^{n}} q \int_{M} \psi(u) u^{2} d \mu_{g} .
$$

By Lemma 5 we deduce that

$$
\frac{1}{2} G_{\varepsilon}^{\prime}(u)[\varphi]=\frac{1}{\varepsilon^{n}} \int_{M}\left[2 q \psi(u)-q^{2} \psi^{2}(u)\right] u \varphi d \mu_{g} .
$$

If $u \in H$ is a critical point of $I_{\varepsilon}$ then the pair $(u, \psi(u))$ is the desired solution of Problem (1) or (2). 


\section{The NEHARI MANIFOLD}

It is well known that a critical point of the free functional $I_{\varepsilon}(u)$ can be found as a critical point constrained on the natural constraint

$$
\mathcal{N}_{\varepsilon}=\left\{u \in H \backslash\{0\}: I_{\varepsilon}^{\prime}(u) u=0\right\} .
$$

We want to prove that the Nehari manifold $\mathcal{N}_{\varepsilon}$ is a $C^{2}$ manifold when $p \geq 4$. (Here is the only point in which for Schroedinger Maxwell systems we require $p>4$ ).

Lemma 6. It holds that

(1) $\mathcal{N}_{\varepsilon}$ is a $C^{2}$ manifold and $\inf _{\mathcal{N}_{\varepsilon}}\|u\|_{\varepsilon}>0$.

(2) It holds the Palais-Smale condition for the functional $I_{\varepsilon \mid \mathcal{N}_{\varepsilon}}$ on $\mathcal{N}_{\varepsilon}$ and for the functional $I_{\varepsilon \mid \mathcal{N}_{\varepsilon}}$ on $H$.

(3) For all $u \in H$ such that $\left|u^{+}\right|_{\varepsilon, p}=1$ there exists a unique positive number $t_{\varepsilon}=t_{\varepsilon}(u)$ such that $t_{\varepsilon}(u) u \in \mathcal{N}_{\varepsilon}$. Moreover $t_{\varepsilon}(u)$ depends continuously on $u$, provided that $u^{+} \not \equiv 0$.

(4) $\lim _{\varepsilon \rightarrow 0} t_{\varepsilon}\left(Z_{\varepsilon, \xi}\right)=1$ uniformly with respect to $\xi \in \partial M$

The proof of this lemma is postponed in the appendix.

Remark 7. We notice that, if $u \in \mathcal{N}_{\varepsilon}$, then

$$
\begin{aligned}
I_{\varepsilon}(u) & =\left(\frac{1}{2}-\frac{1}{p}\right)\|u\|_{\varepsilon}^{2}+\left(\frac{1}{2}-\frac{2}{p}\right) \frac{\omega^{2} q}{\varepsilon^{n}} \int_{M} u^{2} \psi(u) d \mu_{g}+\frac{\omega^{2} q^{2}}{\varepsilon^{n} p} \int_{M} u^{2} \psi^{2}(u) d \mu_{g} \\
& =\left(\frac{1}{2}-\frac{1}{p}\right)\left|u^{+}\right|_{p, \varepsilon}^{p}+\frac{1}{2} \frac{\omega^{2} q^{2}}{\varepsilon^{n}} \int_{M} u^{2} \psi^{2}(u) d \mu_{g}-\frac{1}{2} \frac{\omega^{2} q}{\varepsilon^{n}} \int_{M} u^{2} \psi(u) d \mu_{g}
\end{aligned}
$$

Definition 8. We define

$$
m_{\varepsilon}:=\inf \left\{I_{\varepsilon}(u): u \in \mathcal{N}_{\varepsilon}\right\} .
$$

\section{Strategy of the proof of Theorem 1}

We sketch the proof of our main result. First of all, since the functional $I_{\varepsilon} \in C^{2}$ is bounded below and satisfies PS condition on the manifold $\mathcal{N}_{\varepsilon}$, we have, by well known Lusternik Schnirelmann theorem, that $I_{\varepsilon}$ has at least cat $I_{\varepsilon}^{d}$ critical points in the sublevel

$$
I_{\varepsilon}^{d}=\left\{u \in \mathcal{N}_{\varepsilon}: I_{\varepsilon}(u) \leq d\right\} .
$$

We prove that, for $\varepsilon$ and $\delta$ small enough, it holds

$$
\operatorname{cat} \partial M \leq \operatorname{cat}\left(\mathcal{N}_{\varepsilon} \cap I_{\varepsilon}^{m_{e}^{+}+\delta}\right)
$$

where $m_{e}^{+} \in \mathbb{R}$ will be defined in Section 5 (Proposition 9)

To get (16) we build two continuous operators

$$
\begin{aligned}
& \Phi_{\varepsilon}: \partial M \rightarrow \mathcal{N}_{\varepsilon} \cap I_{\varepsilon}^{m_{e}^{+}+\delta} \\
& \beta: \mathcal{N}_{\varepsilon} \cap I_{\varepsilon}^{m_{e}^{+}+\delta} \rightarrow(\partial M)_{2 \rho}
\end{aligned}
$$

where $(\partial M)_{2 \rho}=\left\{x \in \mathbb{R}^{N}: d(x, \partial M)<2 \rho\right\}$ with $\rho$ small enough in order to have $\operatorname{cat} \partial M \leq \operatorname{cat}(\partial M)_{2 \rho}$.

We build these operators $\Phi_{\varepsilon}$ and $\beta$ such that $\beta \circ \Phi_{\varepsilon}: \partial M \rightarrow(\partial M)_{2 \rho}$ is homotopic to the immersion $i: \partial M \rightarrow(\partial M)_{2 \rho}$. Thus, by the properties of Lusternik Schinrelmann category we obtain (16). Then applying the above mentioned Lusternik Schnirelmann theorem we obtain the proof of our main result. 


\section{The MAP $\Phi_{\varepsilon}$}

We define a function

$$
\begin{gathered}
\Phi_{\varepsilon}: \partial M \rightarrow \mathcal{N}_{\varepsilon} \\
\Phi_{\varepsilon}(q)=t_{\varepsilon}\left(Z_{\varepsilon, \xi}\right) Z_{\varepsilon, \xi}
\end{gathered}
$$

Proposition 9. For any $\varepsilon>0$ the application $\Phi_{\varepsilon}: \partial M \rightarrow \mathcal{N}_{\varepsilon}$ is continuous. Moreover, for any $\delta>0$ there exists $\varepsilon_{0}=\varepsilon_{0}(\delta)>0$ such that, if $\varepsilon<\varepsilon_{0}$ then

$$
\Phi_{\varepsilon}(\xi) \in \mathcal{N}_{\varepsilon} \cap J_{\varepsilon}^{m_{e}^{+}+\delta} \text { for all } \xi \in \partial M
$$

being

$$
\begin{aligned}
m_{e}^{+} & =\inf \left\{E^{+}(v): v \in \mathcal{N}\left(E^{+}\right)\right\} \\
E^{+}(v) & =\int_{\mathbb{R}_{+}^{n}} \frac{1}{2}|\nabla v|^{2}+\frac{\left(a-\omega^{2}\right)}{2}|v|^{2}-\frac{1}{p}\left|v^{+}\right|^{p} d x ; \\
\mathcal{N}\left(E^{+}\right) & =\left\{v \in H^{1}\left(\mathbb{R}_{+}^{n}\right) \backslash\{0\}: E^{+}(v) v=0\right\} ;
\end{aligned}
$$

Proof. The continuity follows directly by the continuity of $t_{\varepsilon}$. For the second claim, we observe that

$$
I_{\varepsilon}\left(t_{\varepsilon}\left(Z_{\varepsilon, \xi}\right) Z_{\varepsilon, \xi}\right)=\frac{1}{2} t_{\varepsilon}^{2}\left\|Z_{\varepsilon, \xi}\right\|_{\varepsilon}^{2}-\frac{1}{p} t_{\varepsilon}^{p}\left|Z_{\varepsilon, \xi}\right|_{\varepsilon, p}^{p}+\frac{1}{\varepsilon^{n}} q t_{\varepsilon}^{2} \int_{M} \psi\left(t_{\varepsilon} Z_{\varepsilon, \xi}\right) Z_{\varepsilon, \xi} d \mu_{g}
$$

In light of Lemma [6 claim 4, we have that $t_{\varepsilon}\left(Z_{\varepsilon, \xi}\right) \rightarrow 1$ as $\varepsilon \rightarrow 0$, uniformly with respect to $\xi \in \partial M$. Moreover, since $t_{\varepsilon}\left(Z_{\varepsilon, \xi}\right) \rightarrow 1$ and by (42) have, uniformly with respect to $\xi$,

Finally, by Remark 21, we get

$$
\frac{1}{\varepsilon^{n}} q t_{\varepsilon}^{2} \int_{M} \psi\left(t_{\varepsilon} Z_{\varepsilon, \xi}\right) Z_{\varepsilon, \xi} d \mu_{g} \rightarrow 0
$$

$$
\lim _{\varepsilon \rightarrow 0} I_{\varepsilon}\left(t_{\varepsilon}\left(Z_{\varepsilon, q}\right) Z_{\varepsilon, q}\right)=\frac{1}{2} \int_{\mathbb{R}_{+}^{n}}|\nabla V|^{2}+\left(a-\omega^{2}\right) V^{2} d y-\frac{1}{p} \int_{\mathbb{R}_{+}^{n}} V^{p} d y=m_{e}^{+}
$$

uniformly with respect to $q \in \partial M$.

Remark 10. By Proposition 9, given $\delta$, we have that $\mathcal{N}_{\varepsilon} \cap J_{\varepsilon}^{m_{e}^{+}+\delta} \neq \emptyset$ for $\varepsilon$ small enough. Moreover we have

$$
\limsup _{\varepsilon \rightarrow 0} m_{\varepsilon} \leq m_{e}^{+} .
$$

\section{Concentration Results}

For any $\varepsilon>0$ we can construct a finite closed partition $\mathcal{P}^{\varepsilon}=\left\{P_{j}^{\varepsilon}\right\}_{j \in \Lambda_{\varepsilon}}$ of $M$ such that

- $P_{j}^{\varepsilon}$ is closed for every $j$ and $P_{j}^{\varepsilon} \cap P_{k}^{\varepsilon} \subset \partial P_{j}^{\varepsilon} \cap \partial P_{k}^{\varepsilon}$ for $j \neq k$;

- $K_{1} \varepsilon \leq d_{j}^{\varepsilon} \leq K_{2} \varepsilon$, where $d_{j}^{\varepsilon}$ is the diameter of $P_{j}^{\varepsilon}$ and $c_{1} \varepsilon^{n} \leq \mu_{g}\left(P_{j}^{\varepsilon}\right) \leq$ $c_{2} \varepsilon^{n}$

- for any $j$ there exists an open set $I_{j}^{\varepsilon} \supset P_{j}^{\varepsilon}$ such that, if $P_{j}^{\varepsilon} \cap \partial M=\emptyset$, then $d_{g}\left(I_{j}^{\varepsilon}, \partial M\right)>K \varepsilon / 2$, while, if $P_{j}^{\varepsilon} \cap \partial M \neq \emptyset$, then $I_{j}^{\varepsilon} \subset\left\{x \in M: d_{g}(x, \partial M) \leq \frac{3}{2} K \varepsilon\right\} ;$

- there exists a finite number $\nu(M) \in \mathbb{N}$ such that every $x \in M$ is contained in at most $\nu(M)$ sets $I_{j}^{\varepsilon}$, where $\nu(M)$ does not depends on $\varepsilon$.

By compactness of $M$ such a partition exists, at least for small $\varepsilon$. In the following we will choose always $\varepsilon_{0}(\delta)$ sufficiently small in order to have this partition. 
Lemma 11. There exists a constant $\gamma>0$ such that, for any fixed $\delta>0$ and for any $\varepsilon \in\left(0, \varepsilon_{0}(\delta)\right)$, where $\varepsilon_{0}(\delta)$ is as in Proposition 9, given any partition $\mathcal{P}^{\varepsilon}$ of $M$ as above, and any function $u \in \mathcal{N}_{\varepsilon} \cap J_{\varepsilon}^{m_{e}^{+}+\delta}$, there exists a set $P_{j}^{\varepsilon} \subset \mathcal{P}^{\varepsilon}$ such that

$$
\frac{1}{\varepsilon^{n}} \int_{P_{j}^{\varepsilon}}\left|u^{+}\right|^{p} d \mu_{g} \geq \gamma>0
$$

Proof. By Remark 10 we have that $\mathcal{N}_{\varepsilon} \cap J_{\varepsilon}^{m_{e}^{+}+\delta} \neq \emptyset$. For any function $u \in \mathcal{N}_{\varepsilon} \cap$ $J_{\varepsilon}^{m_{e}^{+}+\delta}$ we denote by $u_{j}^{+}$the restriction of $u^{+}$to the set $P_{j}^{\varepsilon}$. Then we can write

$$
\begin{aligned}
\|u\|_{\varepsilon}^{2} & =\frac{1}{\varepsilon^{n}} \int_{M}\left(u^{+}\right)^{p} d \mu_{g}-\frac{q \omega^{2}}{\varepsilon^{3}} \int_{M}(2-q \psi(u)) \psi(u) u^{2} d \mu_{g} \\
& \leq \frac{1}{\varepsilon^{n}} \int_{M}\left(u^{+}\right)^{p} d \mu_{g}=\frac{1}{\varepsilon^{n}} \sum_{j} \int_{M}\left(u_{j}^{+}\right)^{p} d \mu_{g}= \\
& =\sum_{j} \frac{\left|u_{j}^{+}\right|_{p}^{p-2}}{\varepsilon^{\frac{n(p-2)}{p}}} \frac{\left|u_{j}^{+}\right|_{p}^{2}}{\varepsilon^{\frac{2 n}{p}}} \leq \max _{j}\left\{\frac{\left|u_{j}^{+}\right|_{p}^{p-2}}{\varepsilon^{\frac{n(p-2)}{p}}}\right\} \sum_{j} \frac{\left|u_{j}^{+}\right|_{p}^{2}}{\varepsilon^{\frac{2 n}{p}}} .
\end{aligned}
$$

Then the proof follows exactly as in [12], Lemma 5.1.

Remark 12. Fixed $\delta$ and $\varepsilon$, we recall that the Ekeland variational principle states that, for any $u \in \mathcal{N}_{\varepsilon} \cap J_{\varepsilon}^{m_{\varepsilon}+2 \delta}$ there exists $u_{\delta} \in \mathcal{N}_{\varepsilon}$ such that

$$
\begin{gathered}
I_{\varepsilon}\left(u_{\delta}\right)<I_{\varepsilon}(u), \quad\left\|u_{\delta}-u\right\|_{\varepsilon}<4 \sqrt{\delta} ; \\
\left|\left(I_{\varepsilon \mid \mathcal{N}_{\varepsilon}}\right)^{\prime}\left(u_{\delta}\right)[\varphi]\right|<\sqrt{\delta}\|\varphi\|_{\varepsilon} .
\end{gathered}
$$

Moreover, since a Palais Smale sequence for $I_{\varepsilon \mid \mathcal{N}_{\varepsilon}}$ is indeed a PS sequence for the free functional we have also that

$$
\left|I_{\varepsilon}^{\prime}\left(u_{\delta}\right)[\varphi]\right|<\sqrt{\delta}\|\varphi\|_{\varepsilon} .
$$

Proposition 13. For all $\eta \in(0,1)$ there exists a $\delta_{0}<m_{e}^{+}$such that for any $\delta \in\left(0, \delta_{0}\right)$ for any $\varepsilon \in\left(0, \varepsilon_{0}(\delta)\right.$ ) (as in Prop. 9) and for any function $u \in \mathcal{N}_{\varepsilon} \cap I_{\varepsilon}^{m_{e}^{+}+\delta}$ we can find a point $\xi=\xi(u) \in \partial M$ for which

$$
\left(\frac{1}{2}-\frac{1}{p}\right) \frac{1}{\varepsilon^{n}} \int_{I_{\xi}(\rho, R)}\left|u^{+}\right|^{p} d \mu_{g} \geq(1-\eta) m_{e}^{+}
$$

Proof. We first prove this property for $u \in \mathcal{N}_{\varepsilon} \cap I_{\varepsilon}^{m_{e}^{+}+\delta} \cap I_{\varepsilon}^{m_{\varepsilon}+2 \delta}$.

Assume, by contradiction, that there exists $\eta \in(0,1)$, two sequences of vanishing real numbers $\left\{\delta_{k}\right\}_{k}$ and $\left\{\varepsilon_{k}\right\}_{k}$ and a sequence of functions $\left\{u_{k}\right\}_{k} \subset \mathcal{N}_{\varepsilon_{k}} \cap I_{\varepsilon_{k}}^{m_{e}^{+}+\delta_{k}} \cap$ $I_{\varepsilon_{k}}^{m_{\varepsilon_{k}}+2 \delta_{k}}$ such that, for any $\xi \in \partial M$ it holds

$$
\left(\frac{1}{2}-\frac{1}{p}\right) \frac{1}{\varepsilon_{k}^{n}} \int_{I_{\xi}(\rho, R)}\left|u_{k}^{+}\right|^{p} d \mu_{g}<(1-\eta) m_{e}^{+} .
$$

By Remark 12 we can assume

$$
J_{\varepsilon_{k}}^{\prime}\left(u_{k}\right)[\varphi] \leq \sqrt{\delta_{k}}\|\varphi\|_{\varepsilon_{k}} \text { for all } \varphi \in H_{g}^{1}(M)
$$


By Lemma 11 there exists a set $P_{k}^{\varepsilon_{k}} \in \mathcal{P}_{\varepsilon_{k}}$ such that

$$
\frac{1}{\varepsilon_{k}^{n}} \int_{P_{k}^{\varepsilon_{k}}}\left|u_{k}^{+}\right|^{p} d \mu_{g} \geq \gamma>0 .
$$

we have to examine two cases: either there exists a subsequence $P_{i_{k}}^{\varepsilon_{i_{k}}}$ such that $P_{i_{k}}^{\varepsilon_{i_{k}}} \cap \partial M \neq \emptyset$, or there exists a subsequence $P_{i_{k}}^{\varepsilon_{i_{k}}}$ such that $P_{i_{k}}^{\varepsilon_{i_{k}}} \cap \partial M=\emptyset$. For simplicity we write simply $P_{k}$ for $P_{i_{k}}^{\varepsilon_{i_{k}}}$.

First case: $P_{k} \cap \partial M \neq \emptyset$. We choose a point $\xi_{k}$ interior to $P_{k} \cap \partial M$. We have the Fermi coordinates $\Psi_{\xi_{k}}^{\partial}: B_{n-1}(0, R) \times[0, R] \rightarrow M, \Psi_{\xi_{k}}^{\partial}\left(\bar{y}, y_{n}\right)=\left(\bar{x}, x_{n}\right)=x$, being $B_{n-1}(0, R)=\left\{\bar{y} \in \mathbb{R}^{n-1},|\bar{y}|<R\right\}$. In what follows we simply call

$$
B(R)_{+}:=B_{n-1}(0, R) \times[0, R]
$$

We consider the function $w_{k}: \mathbb{R}_{+}^{n} \rightarrow \mathbb{R}$ defined by

$$
u_{k}\left(\Psi_{\xi_{k}}^{\partial}\left(\bar{y}, y_{n}\right)\right) \chi_{R}(|\bar{y}|) \chi_{R}\left(y_{n}\right)=u_{k}\left(\Psi_{\xi_{k}}^{\partial}\left(\varepsilon_{k} \bar{z}, \varepsilon z_{n}\right)\right) \chi_{R}\left(\left|\varepsilon_{k} \bar{z}\right|\right) \chi_{R}\left(\varepsilon z_{n}\right)=w_{k}\left(\bar{z}, z_{n}\right) .
$$

It is clear that $w_{k} \in H^{1}\left(\mathbb{R}_{+}^{n}\right)$ with $w_{k}\left(\bar{z}, z_{n}\right)=0$ when $|\bar{z}|=0, R / \varepsilon_{k}$ or $z_{n}=R / \varepsilon_{k}$. We now show some properties of the function $w_{k}$.

It is easy to see (cfr. [12, Prop. 5.3) that $\left\{w_{k}\right\}_{k}$ is bounded in $H^{1}\left(\mathbb{R}_{+}^{n}\right)$. Then there exists $w \in H^{1}\left(\mathbb{R}_{+}^{n}\right)$ such that $w_{k}$ converges to $w$ weakly in $H^{1}\left(\mathbb{R}_{+}^{n}\right)$ and strongly in $L_{\text {loc }}^{p}\left(\mathbb{R}_{+}^{n}\right)$.

We claim that the limit function $w$ is a weak solution of

$$
\left\{\begin{array}{cc}
-\Delta w+\left(a-\omega^{2}\right) w=\left(w^{+}\right)^{p-1} & \text { in } \mathbb{R}_{+}^{n} ; \\
\frac{\partial w}{\partial \nu}=0 & \text { for } y=(\bar{y}, 0)
\end{array}\right.
$$

First, for any $f \in C_{0}^{\infty}\left(\mathbb{R}_{+}^{n}\right)$ we define on the manifold $M$ the function

$$
f_{k}(x):=f\left(\frac{1}{\varepsilon_{k}}\left(\Psi_{\xi_{k}}^{\partial}\right)^{-1}(x)\right)=f(z) \text { where } x=\Psi_{\xi_{k}}^{\partial}\left(\varepsilon_{k} z\right) .
$$

We notice that for every $f \in C_{0}^{\infty}\left(\mathbb{R}^{3}\right)$, there exists $k$ such that $\operatorname{supp} f \subset B\left(0, R / 2 \varepsilon_{k}\right)$. Thus, $\operatorname{supp} f_{k} \subset I_{\xi_{k}}(R / 2)$.

Moreover, we have $\left\|f_{k}\right\|_{\varepsilon_{k}} \leq C\|f\|_{H^{1}\left(\mathbb{R}^{3}\right)}$, thus, by Ekeland principle we have

$$
\left|I_{\varepsilon_{k}}^{\prime}\left(u_{k}\right)\left[f_{k}\right]\right| \leq \sigma_{k}\left\|f_{k}\right\|_{\varepsilon_{k}} \rightarrow 0 \text { while } k \rightarrow \infty .
$$

On the other hand we have

$$
\begin{array}{r}
I_{\varepsilon}^{\prime}\left(u_{k}\right)\left[f_{k}\right]=\frac{1}{\varepsilon_{k}^{n}} \int_{M} \varepsilon_{k}^{2} \nabla_{g} u_{k} \nabla_{g} f_{k}+a u_{k} f_{k}-\left(u_{k}^{+}\right)^{p-1} f_{k}-\omega^{2}\left(1-q \psi\left(u_{k}\right)\right)^{2} u_{k} f_{k} d \mu_{g} \\
=\left\langle u_{k}, f_{k}\right\rangle_{\varepsilon_{k}}-\frac{1}{\varepsilon_{k}^{n}} \int_{M}\left(u_{k}^{+}\right)^{p-1} f_{k} d \mu_{g}+\frac{q \omega^{2}}{\varepsilon_{k}^{3}} \int_{M}\left(2-q \psi\left(u_{k}\right)\right) \psi\left(u_{k}\right) u_{k} f_{k} d \mu_{g} \\
=\int_{T_{k}}\left[\sum_{i j} g_{\xi_{k}}^{i j}\left(\varepsilon_{k} z\right) \partial_{z_{i}} w_{k}(z) \partial_{z_{j}} f(z)+\left(a-\omega^{2}\right) w_{k}(z) f(z)\right]\left|g_{\xi_{k}}(\varepsilon z)\right|^{1 / 2} d z \\
-\int_{T_{k}}\left(w_{k}^{+}(z)\right)^{p-1} f(z)\left|g_{\xi_{k}}(\varepsilon z)\right|^{1 / 2} d z \\
+q \omega^{2} \int_{T_{k}}\left(2-q \tilde{\psi}_{k}(z)\right) \tilde{\psi}_{k}(z) w_{k}(z) f(z)\left|g_{\xi_{k}}(\varepsilon z)\right|^{1 / 2} d z
\end{array}
$$


Here $T_{k}=B\left(R / 2 \varepsilon_{k}\right)_{+} \cap \operatorname{supp} f$ and $\psi\left(u_{k}\right)(x):=\psi_{k}(x)=\psi_{k}\left(\Psi_{\xi_{k}}^{\partial}\left(\varepsilon_{k} z\right)\right):=\tilde{\psi}_{k}(z)$ where $x \in I_{\xi_{k}}(R)$ and $z \in B\left(R / \varepsilon_{k}\right)_{+}$. Since $\operatorname{supp} f_{k} \subset I_{\xi_{k}}(R / 2)$, for KGMP systems, by (10) we have

$$
\begin{aligned}
0= & \int_{M} \nabla_{g} \psi\left(u_{k}\right) \nabla_{g} f_{k}+\left(1+q^{2} u_{k}\right) \psi\left(u_{k}\right) f_{k}-q u_{k}^{2} f_{k} d \mu_{g} \\
= & \frac{\varepsilon_{k}^{3}}{\varepsilon_{k}^{2}} \int_{T_{k}} \sum_{i j} g_{q_{k}}^{i j}\left(\varepsilon_{k} z\right) \partial_{z_{i}} \tilde{\psi}_{k}(z) \partial_{z_{j}} f(z)\left|g_{q_{k}}(\varepsilon z)\right|^{1 / 2} d z \\
& +\varepsilon_{k}^{3} \int_{T_{k}}\left(1+q^{2} w_{k}(z)\right) \tilde{\psi}_{k}(z) f(z)\left|g_{q_{k}}(\varepsilon z)\right|^{1 / 2} d z \\
& -\varepsilon_{k}^{3} \int_{T_{k}} q w_{k}^{2}(z) f(z)\left|g_{q_{k}}(\varepsilon z)\right|^{1 / 2} d z,
\end{aligned}
$$

The above equation holds for KGMP systems but the analogous for KGM systems is obvious. We have

$$
\begin{aligned}
& -\int_{T_{k}} \sum_{i j} g_{\xi_{k}}^{i j}\left(\varepsilon_{k} z\right) \partial_{z_{i}} \tilde{\psi}_{k}(z) \partial_{z_{j}} f(z)\left|g_{\xi_{k}}(\varepsilon z)\right|^{1 / 2} d z= \\
& \quad=\varepsilon_{k}^{2} \int_{T_{k}}\left(\left(1+q^{2} w_{k}(z)\right) \tilde{\psi}_{k}(z)-q w_{k}^{2}(z)\right) f(z)\left|g_{\xi_{k}}(\varepsilon z)\right|^{1 / 2} d z
\end{aligned}
$$

Arguing as in Lemma 19 we have that

$$
\begin{aligned}
c \int_{B\left(R / \varepsilon_{k}\right)_{+}}\left|\nabla \tilde{\psi}_{k}(z)\right|^{2} d z & \leq \frac{\varepsilon_{k}^{2}}{\varepsilon_{k}^{n}} \int_{M}\left|\nabla_{g} \psi_{k}\right|^{2} d \mu_{g} \leq \frac{1}{\varepsilon_{k}^{n}} q \int_{M} u_{k}^{2} \psi_{k} \\
& \leq \frac{1}{\varepsilon_{k}^{n}} \int u_{k}^{2} \leq\left\|u_{k}\right\|_{\varepsilon_{k}}^{2} \leq C
\end{aligned}
$$

where $c, C>0$ are suitable constants. Moreover, by Lemma 19]

$$
\begin{aligned}
c_{1} \int_{B\left(0, R / \varepsilon_{k}\right)}\left|\tilde{\psi}_{k}(z)\right|^{2} d z & \leq \frac{1}{\varepsilon_{k}^{n}} \int_{M} \psi_{k}^{2} d \mu_{g} \leq \frac{1}{\varepsilon_{k}^{n}}\left\|\psi_{k}\right\|_{H_{g}^{1}}^{2} \leq c_{2} \frac{1}{\varepsilon_{k}^{n}}\left|u_{k}\right|_{4, g}^{4} \\
& \leq c_{2}\left|u_{k}\right|_{4, \varepsilon}^{4} \leq C
\end{aligned}
$$

where $c_{1}, c_{2}, C>0$ are suitable constants. Conlcuding, we have that $\left\|\tilde{\psi}_{k}\right\|_{H^{1}\left(B\left(R / \varepsilon_{k}\right)_{+}\right)}$ is bounded, and then also $\left\|\chi_{R / \varepsilon_{k}}(z) \tilde{\psi}_{k}(z)\right\|_{H^{1}\left(\mathbb{R}_{+}^{n}\right)}^{2}$ is bounded. So, there exists a $\bar{\psi} \in H^{1}\left(\mathbb{R}_{+}^{n}\right)$ such that $\bar{\psi}_{k}(z):=\chi_{R / \varepsilon_{k}}(z) \tilde{\psi}_{k}(z) \rightarrow \bar{\psi}$ weakly in $H^{1}\left(\mathbb{R}_{+}^{n}\right)$ and strongly in $L_{\mathrm{loc}}^{p}\left(\mathbb{R}_{+}^{n}\right)$ for any $2 \leq p<2^{*}$.

By (22) we have

$$
\begin{aligned}
-\int_{\mathbb{R}_{+}^{n}} \sum_{i j} g_{\xi_{k}}^{i j}\left(\varepsilon_{k} z\right) \partial_{z_{i}} \bar{\psi}_{k}(z) \partial_{z_{j}} f(z)\left|g_{\xi_{k}}(\varepsilon z)\right|^{1 / 2} d z= \\
=\varepsilon_{k}^{2} \int_{\mathbb{R}_{+}^{n}}\left(\left(1+q^{2} w_{k}(z)\right) \bar{\psi}_{k}(z)-q w_{k}^{2}(z)\right) f(z)\left|g_{\xi_{k}}(\varepsilon z)\right|^{1 / 2} d z
\end{aligned}
$$

and, using that $g_{k}^{i j}(\varepsilon z)=\delta_{i j}+O\left(\varepsilon_{k}|z|\right)$ and that $\left|g_{q}(\varepsilon z)\right|^{1 / 2}=1+O\left(\varepsilon_{k}|z|\right)$ we get

$$
\int_{\mathbb{R}_{+}^{n}} \nabla \bar{\psi}_{k}(z) \nabla f(z) d z=O\left(\varepsilon_{k}\right) .
$$

Thus, the function $\bar{\psi} \in H^{1}\left(\mathbb{R}_{+}^{n}\right)$ is a weak solution of $-\Delta \bar{\psi}=0$, so $\bar{\psi}=0$. 
At this point, arguing as above we have

$$
\begin{aligned}
\frac{1}{\varepsilon_{k}^{n}} \int_{M}\left(2-q \psi\left(u_{k}\right)\right) \psi\left(u_{k}\right) u_{k} f_{k} d \mu_{g} & =\frac{1}{\varepsilon_{k}^{n}} \int_{I_{\xi_{k}}(R / 2)}\left(2-q \psi\left(u_{k}\right)\right) \psi\left(u_{k}\right) u_{k} f_{k} d \mu_{g}= \\
& =\int_{\operatorname{supp} f}\left(2-q \bar{\psi}_{k}\right) \bar{\psi}_{k} w_{k} f\left|g_{\xi_{k}}(\varepsilon z)\right|^{1 / 2} d z \rightarrow 0
\end{aligned}
$$

while $k \rightarrow \infty$ because $\bar{\psi}_{k} \rightarrow 0$ strongly in $L_{\text {loc }}^{p}\left(\mathbb{R}_{+}^{n}\right)$ for any $2 \leq p<2^{*}$. Thus, by (23), (20) and (21) and because $w_{k} \rightarrow w$ in $H^{1}$ we deduce that, for any $f \in C_{0}^{\infty}\left(\mathbb{R}^{3}\right)$, it holds

$$
\int_{\mathbb{R}_{+}^{n}} \nabla w \nabla f+\left(a-\omega^{2}\right) w f-\left(w^{+}\right)^{p-1} f=0 .
$$

Thus, $w$ is a weak solution of $-\Delta w+\left(a-\omega^{2}\right) w=w^{p-1}$ on $\mathbb{R}_{+}^{n}$ with Neumann boundary condition. Since $u_{k} \in \mathcal{N}_{\varepsilon_{k}} \cap I_{\varepsilon_{k}}^{m_{e}^{+}+\delta_{k}}$ we have

$$
\left(\frac{1}{2}-\frac{1}{p}\right)\left\|u_{k}\right\|_{\varepsilon_{k}}^{2} \leq I_{\varepsilon_{k}}\left(u_{k}\right) \leq m_{e}^{+}+\delta_{k},
$$

hence

$$
\|w\|_{a}^{2} \leq \liminf _{k}\left\|w_{k}\right\|_{a}^{2} \leq \frac{2 p}{p-2} m_{e}^{+}
$$

where $\|w\|_{a}^{2}=\frac{1}{2} \int_{\mathbb{R}_{+}^{n}}|\nabla w|^{2}+\left(a-\omega^{2}\right) u^{2}$. Set

$$
\mathcal{N}_{\infty}=\left\{v \in H^{1}\left(\mathbb{R}_{+}^{n}\right) \backslash\{0\}:\|v\|_{a}^{2}=|v|_{p}^{p}\right\},
$$

we have that $w \in \mathcal{N}_{\infty} \cup\{0\}$. Since $P_{k} \cap \partial M \neq \emptyset$, we can choose $T>0$ such that

$$
P_{k} \subset I_{\xi_{k}}\left(\varepsilon_{k} T, \varepsilon_{k} T\right) \text { for } k \text { large enough. }
$$

for $\xi_{k} \in P_{k} \cap \partial M$. By definition of $w_{k}$ and by Lemma 11 there exist a $\xi_{k}$ such that, for $k$ large enough

$$
\begin{aligned}
\| w_{k}^{+}(25)_{\left(B_{n-1}(0, T) \times[0, T]\right)} & =\int_{B_{n-1}(0, T) \times[0, T]}\left|\chi_{R}\left(\varepsilon_{k}|\bar{z}|\right) \chi_{\rho}\left(\varepsilon_{k} z_{n}\right) u_{k}^{+}\left(\psi_{q_{k}}^{\partial}\left(\varepsilon_{k} z\right)\right)\right|^{p} d z= \\
& =\frac{1}{\varepsilon_{k}^{n}} \int_{B_{n-1}\left(0, \varepsilon_{k} T\right) \times\left[0, \varepsilon_{k} T\right]}\left|u_{k}^{+}\left(\psi_{q_{k}}^{\partial}(y)\right)\right|^{p} d y \geq \\
& \geq \frac{c}{\varepsilon_{k}^{n}} \int_{B_{n-1}\left(0, \varepsilon_{k} T\right) \times\left[0, \varepsilon_{k} T\right]}\left|u_{k}^{+}\left(\psi_{q_{k}}^{\partial}(y)\right)\right|^{p}\left|g_{q_{k}}(y)\right|^{1 / 2} d y= \\
& \geq \frac{c}{\varepsilon_{k}^{n}} \int_{I_{q_{k}}\left(\varepsilon_{k} T, \varepsilon_{k} T\right)}\left|u_{k}^{+}\right|^{p} d \mu_{g} \geq c \gamma>0 .
\end{aligned}
$$

Since $w_{k}$ converge strongly to $w$ in $L^{p}\left(B_{n-1}(0, T) \times[0, T]\right)$, we have $w \neq 0$, so $w \in \mathcal{N}_{\infty}$. Hence, by (24) we obtain that

$$
\|w\|_{a}^{2}=|w|_{p}^{p}=\frac{2 p}{p-2} m_{e}^{+}
$$

and that $w_{k} \rightarrow w$ strongly in $H^{1}\left(\mathbb{R}_{+}^{n}\right)$. From this we derive the contradiction. Indeed, since $\left|g_{q}\left(\varepsilon_{k} z\right)\right|^{1 / 2}=1+O\left(\varepsilon_{k}|z|\right)$, fixed $T$, by (18), for $k$ large it holds

$$
\int_{B(T)_{+}}\left(w_{k}^{+}\right)^{p} d z \leq\left(1-\frac{\eta}{2}\right) \frac{2 p}{p-2} m_{\infty} .
$$


Moreover, by (26) there exists a $T>0$ such that $\int_{B(T)_{+}} w^{p} d z>\left(1-\frac{\eta}{8}\right) \frac{2 p}{p-2} m_{\infty}$ and, since $w_{k} \rightarrow w$ strongly in $L_{\mathrm{loc}}^{p}\left(\mathbb{R}_{+}^{n}\right), \int_{B(T)_{+}}\left(w_{k}^{+}\right)^{p} d z>\left(1-\frac{\eta}{4}\right) \frac{2 p}{p-2} m_{\infty}$, that contradicts (27).

Second case: $P_{k}^{\varepsilon} \cap \partial M=\emptyset$. In this case we choose a point $\xi_{k}$ interior to $P_{k}^{\varepsilon}$ and we consider the normal coordinates at $\xi_{k}$. We set $w_{k}(z)$ as

$$
u_{k}(x) \chi_{R}\left(\exp _{\xi_{k}}^{-1}(x)\right)=u_{k}\left(\exp _{\xi_{k}}(y)\right) \chi_{R}(y)=u_{k}\left(\exp _{\xi_{k}}\left(\varepsilon_{k} z\right)\right) \chi_{R}\left(\varepsilon_{k} z\right)=w_{k}(z) .
$$

Arguing as in the previous case, we can establish that $w_{k}$ is bounded in $H^{1}\left(\mathbb{R}^{n}\right)$ and converges to some $w \in H^{1}\left(\mathbb{R}^{n}\right)$ weakly in $H^{1}\left(\mathbb{R}^{n}\right)$ and strongly in $L_{\text {loc }}^{p}\left(\mathbb{R}^{n}\right)$. Moreover $w \neq 0$ and is a solution of $-\Delta w+\left(a-\omega^{2}\right) w=w^{p-1}$ in $\mathbb{R}^{n}$. Thus $\|w\|_{a}^{2}=|w|_{p}^{p}=2 \frac{2 p}{p-2} m_{e}^{+}$and $w_{k} \rightarrow w$ strongly in $H^{1}\left(\mathbb{R}^{n}\right)$ and from this follows the contradiction.

Conclusion: We have proved the claim for $u_{k} \in \mathcal{N}_{\varepsilon_{k}} \cap I_{\varepsilon_{k}}^{m_{e}^{+}+\delta_{k}} \cap I_{\varepsilon_{k}}^{m_{\varepsilon}+2 \delta_{k}}$. We prove now the claim in the general case. For $u_{k}$ it holds

$$
\begin{aligned}
I_{\varepsilon_{k}}\left(u_{k}\right) & =\left(\frac{1}{2}-\frac{1}{p}\right)\left|u_{k}^{+}\right|_{p, \varepsilon_{k}}^{p}+\frac{1}{2} \frac{\omega^{2} q^{2}}{\varepsilon_{k}^{n \grave{l}}} \int_{M} u_{k}^{2} \psi^{2}\left(u_{k}\right) d \mu_{g}-\frac{1}{2} \frac{\omega^{2} q}{\varepsilon_{k}^{n}} \int_{M} u_{k}^{2} \psi\left(u_{k}\right) d \mu_{g} \\
& \geq(1-\eta) m_{e}^{+}-\frac{1}{2} \frac{\omega^{2} q}{\varepsilon_{k}^{3}} \int_{M} u_{k}^{2} \psi\left(u_{k}\right) d \mu_{g}
\end{aligned}
$$

By compactness of $M$ there exists $\xi_{1}, \ldots, \xi_{m} \in M \backslash \partial M$ and $\xi_{m+1}, \ldots, \xi_{l} \in \partial M$ such that

$$
\frac{1}{\varepsilon_{k}^{n}} \int_{M} u_{k}^{2} \psi\left(u_{k}\right) d \mu_{g} \leq \sum_{i=1}^{m} \frac{1}{\varepsilon_{k}^{n}} \int_{B_{g}\left(\xi_{i}, r\right)} u_{k}^{2} \psi\left(u_{k}\right) d \mu_{g}+\sum_{i=m+1}^{l} \frac{1}{\varepsilon_{k}^{n}} \int_{I_{\xi_{i}}(r)} u_{k}^{2} \psi\left(u_{k}\right) d \mu_{g}
$$

For any $\xi_{i}, i=1, \ldots, m$, arguing as above, we can introduce two sequences of functions $w_{k}^{i}$ and $\bar{\psi}_{k}$ such that $w_{k}^{i} \rightarrow w^{i}$, strongly in $H^{1}\left(\mathbb{R}^{n}\right), w^{i}$ solution of $-\Delta w+$ $\left(a-\omega^{2}\right) w=w^{p-1}$, and that $\bar{\psi}_{k}^{i} \rightarrow 0$ strongly in $L_{\mathrm{loc}}^{p}\left(\mathbb{R}^{n}\right)$ for any $2 \leq p<2^{*}$. We thus have that, for any $\xi^{i}$

$$
\frac{1}{\varepsilon_{k}^{n}} \int_{B_{g}\left(\xi^{i}, r\right)} u_{k}^{2} \psi\left(u_{k}\right) d \mu_{g} \leq \int_{\mathbb{R}^{n}}\left(w_{k}^{i}\right)^{2} \bar{\psi}_{k}^{i} d x \rightarrow 0 .
$$

It follows identically, for $i=m+1, \ldots, l$,

$$
\frac{1}{\varepsilon_{k}^{n}} \int_{I_{\xi^{i}}(r)} u_{k}^{2} \psi\left(u_{k}\right) d \mu_{g} \leq \int_{\mathbb{R}_{+}^{n}}\left(w_{k}^{i}\right)^{2} \bar{\psi}_{k}^{i} d x \rightarrow 0 .
$$

Thus $\lim \sup _{k} m_{\varepsilon_{k}} \geq m_{e}^{+}$, and, in light of Remark 10, $\lim _{k} m_{\varepsilon_{k}}=m_{e}^{+}$. Hence, when $\varepsilon, \delta$ are small enough, we have $\mathcal{N}_{\varepsilon} \cap I_{\varepsilon}^{m_{e}^{+}+\delta} \subset \mathcal{N}_{\varepsilon} \cap I_{\varepsilon}^{m_{\varepsilon}+2 \delta}$ and the general claim follows.

\section{The MAP $\beta$}

For any $u \in \mathcal{N}_{\varepsilon}$ with we can define its center of mass as a point $\beta(u) \in \mathbb{R}^{N}$ by

$$
\beta(u)=\frac{\int_{M} x\left|u^{+}(x)\right|^{p} d \mu_{g}}{\int_{M}\left|u^{+}(x)\right|^{p} d \mu_{g}} .
$$

The application is well defined on $\mathcal{N}_{\varepsilon}$, since $u \in \mathcal{N}_{\varepsilon}$ implies $u^{+} \neq 0$ (it follows immediatly by Lemma 6). In the following we will show that if $u \in \mathcal{N}_{\varepsilon} \cap J^{m_{e}^{+}+\delta}$ 
then $\beta(u)$ belong to a tubular neighborhood of $\partial M$, provided $\varepsilon$ and $\delta$ sufficiently small.

Proposition 14. For any $u \in \mathcal{N}_{\varepsilon} \cap J^{m_{e}^{+}+\delta}$, with $\varepsilon$ and $\delta$ small enough, it holds

$$
\beta(u) \in(\partial M)_{3 \rho},
$$

being $(\partial M)_{r}=\left\{x \in \mathbb{R}^{N} d(x, \partial M)<r\right\}$ a neighborhood of $\partial M$ in the space $\mathbb{R}^{N}$ where the manifold $M$ is embedded. Moreover the composition

$$
\beta \circ \Phi_{\varepsilon}: \partial M \rightarrow(\partial M)_{3 \rho}
$$

is well defined and homotopic to the identity of $\partial M$.

Proof. Since $m_{\varepsilon} \rightarrow m_{e}^{+}$and by Proposition 13 we get that for any $u \in \mathcal{N}_{\varepsilon} \cap J^{m_{e}^{+}+\delta}$ there exists $\xi \in \partial M$ such that

$$
(1-\eta) m_{e}^{+} \leq\left(\frac{1}{2}-\frac{1}{p}\right) \frac{1}{\varepsilon^{n}}\left|u^{+}\right|_{L^{p}\left(I_{\xi}(\rho, R)\right)}^{p} .
$$

Since $u \in \mathcal{N}_{\varepsilon} \cap J^{m_{e}^{+}+\delta}$ we have

$$
\begin{aligned}
m_{e}^{+}+\delta & \geq I_{\varepsilon}(u)=\left(\frac{p-2}{2 p}\right)\left|u^{+}\right|_{p, \varepsilon}^{p}+\frac{\omega^{2} q^{2}}{2 \varepsilon^{n}} \int_{M} u^{2} \psi^{2}(u) d \mu_{g}-\frac{\omega^{2} q}{2 \varepsilon^{n}} \int_{M} u^{2} \psi(u) d \mu_{g} \geq \\
& \geq\left(\frac{p-2}{2 p}\right)\left|u^{+}\right|_{p, \varepsilon}^{p}-\frac{\omega^{2} q}{2 \varepsilon^{n}} \int_{M} u^{2} \psi(u) d \mu_{g}
\end{aligned}
$$

Now, arguing as in Lemma 19] we have that, by Holder inequality that $\|\psi(u)\|_{H} \leq$ $\left(\int_{M} u^{12 / 5}\right)^{5 / 6}$, and, in the same way, that

$$
\begin{aligned}
\frac{1}{\varepsilon^{n}} \int_{M} \psi(u) u^{2} & \leq \frac{1}{\varepsilon^{n}}\|\psi\|_{H}\left(\int_{M} u^{12 / 5}\right)^{5 / 6} \leq C \frac{1}{\varepsilon^{n}}\left(\int_{M} u^{12 / 5}\right)^{5 / 3} \\
& \leq C \varepsilon^{\frac{2}{3} n}|u|_{12 / 5, \varepsilon}^{4} \leq C \varepsilon^{\frac{2}{3} n}\|u\|_{\varepsilon}^{4} \leq C \varepsilon^{\frac{2}{3} n}
\end{aligned}
$$

since $\|u\|_{\varepsilon}$ is bounded because $u \in \mathcal{N}_{\varepsilon} \cap I_{\varepsilon}^{m_{\infty}+\delta}$.

So, provided we choose $\varepsilon\left(\delta_{0}\right)$ small enough, we have

$$
\left(\frac{1}{2}-\frac{1}{p}\right) \frac{1}{\varepsilon^{n}}\left|u^{+}\right|_{p, g}^{p}<m_{e}^{+}+2 \delta .
$$

By (29) and (30) we get

$$
\int_{I_{\xi}(\rho, R)} \frac{\left|u^{+}\right|^{p}}{\left|u^{+}\right|_{p, g}^{p}} d \mu_{g} \geq \frac{1-\eta}{1+\frac{2 \delta}{m_{e}^{+}}}
$$

By definition of $\beta$ we have

$$
\begin{aligned}
|\beta(u)-q| & \leq\left|\int_{I_{\xi}(\rho, R)}(x-q) \frac{\left|u^{+}\right|^{p}}{\left|u^{+}\right|_{p, g}^{p}} d \mu_{g}\right|+\left|\int_{M \backslash I_{\xi}(\rho, R)}(x-q) \frac{\left|u^{+}\right|^{p}}{\left|u^{+}\right|_{p, g}^{p}} d \mu_{g}\right| \leq \\
& \leq 2 \rho+D\left(1-\frac{1-\eta}{1+\frac{\delta}{m_{e}^{+}}}\right),
\end{aligned}
$$

where $D$ is the diameter of the manifold $M$ as a subset of $\mathbb{R}^{n}$. Here we supposed, without loss of generality that $R<\rho$. Choosing $\eta$ and $\delta$ small enough we get the first claim. The second claim is standard. 


\section{Profile DESCRIPtion}

Let $u_{\varepsilon}$ a low energy solution. By regularity theory (see [4, Th. 1])we can prove that $u_{\varepsilon} \in C^{\infty}(\bar{M})$. So there exists at least one maximum point of $u_{\varepsilon}$ on $M$. We can prove that, for $\varepsilon$ small, $u_{\varepsilon}$ has a unique local maximum point $P_{\varepsilon} \in \partial M$ and we can describe the profile of $u_{\varepsilon}$.

Lemma 15. Let $\left(u_{\varepsilon}, \psi\left(u_{\varepsilon}\right)\right)$ be solution of (2) such that $I_{\varepsilon}\left(u_{\varepsilon}\right) \leq m_{e}^{+}+\delta<2 m_{e}^{+}$. Then, for $\varepsilon$ small, $u_{\varepsilon}$ is not constant on $M$.

Proof. At first we notice that if $u_{\varepsilon}$ is constant, also $\psi\left(u_{\varepsilon}\right)$ is constant. Moreover, by (2) the values of $u_{\varepsilon}$ and $\psi\left(u_{\varepsilon}\right)$ depend only on $a, \omega, q$ and $p$. Let $u_{\varepsilon}=u_{0}$ and $\psi\left(u_{\varepsilon}\right)=\psi_{0}$. Immediatly we have

$$
\begin{aligned}
I_{\varepsilon}\left(u_{\varepsilon}\right)=\left(\frac{1}{2}-\frac{1}{p}\right) & \frac{1}{\varepsilon^{3}} \int_{M}\left(a-\omega^{2}\right) u_{0}^{2} d \mu_{g} \\
& +\left(\frac{1}{2}-\frac{2}{p}\right) \frac{\omega^{2} q}{\varepsilon^{3}} \int_{M} u_{0}^{2} \psi_{0} d \mu_{g}+\frac{\omega^{2} q^{2}}{\varepsilon^{3} p} \int_{M} u_{0}^{2} \psi_{0}^{2} d \mu_{g} \rightarrow+\infty
\end{aligned}
$$

which leads us to a contradiction.

Since $u_{\varepsilon}$ is not constant and continuous on $\bar{M}$, then there exists at least a maximum point $P \in \bar{M}$. Proceeding as in [13, it is easy to see that if $P \in M \backslash \partial M$ then $I_{\varepsilon}\left(u_{\varepsilon}\right) \geq m_{\infty}=2 m_{\varepsilon}^{+}$where

$$
\begin{aligned}
m_{\infty} & =\inf \{E(v): v \in \mathcal{N}(E)\}=E(U) \text { with } U \text { defined in (5) } \\
E(v) & =\int_{\mathbb{R}^{n}} \frac{1}{2}|\nabla v|^{2}+\frac{\left(a-\omega^{2}\right)}{2}|v|^{2}-\frac{1}{p}\left|v^{+}\right|^{p} d x \\
\mathcal{N}(E) & =\left\{v \in H^{1}\left(\mathbb{R}^{n}\right) \backslash\{0\}: E(v) v=0\right\} .
\end{aligned}
$$

This implies that $P \in \partial M$. Now, since $u_{\varepsilon}$ is regular and $\frac{\partial u}{\partial \nu}=0$ on $\partial M, P$ is also a critical point for $\left.u_{\varepsilon}\right|_{\partial M}$ and $\Delta_{g} u_{\varepsilon}\left(x_{0}\right) \leq 0$. We have the following result.

Lemma 16. Let $P \in \partial M$ be a maximum point for $u_{\varepsilon}$ solution of (2). Then

$$
\left(u_{\varepsilon}(P)\right)^{p-2}>a-\omega^{2}
$$

Proof. We have just pointed out that $\Delta_{g} u_{\varepsilon}(P) \leq 0$. Then

$$
0 \geq \varepsilon^{2} \Delta_{g} u_{\varepsilon}(P)=u_{\varepsilon}(P)\left[a-\left(u_{\varepsilon}(P)\right)^{p-2}-\omega^{2}\left(q \psi\left(u_{\varepsilon}\right)(P)-1\right)^{2}\right]
$$

and, since $\left|q \psi\left(u_{\varepsilon}\right)-1\right|<1$,

$$
a \leq\left(u_{\varepsilon}(P)\right)^{p-2}+\omega^{2}\left(q \psi\left(u_{\varepsilon}\right)(P)-1\right)^{2} \leq\left(u_{\varepsilon}(P)\right)^{p-2}+\omega^{2} .
$$

This ends the proof.

Lemma 17. Let $u_{\varepsilon}$ be a solution of (2) such that $I_{\varepsilon}\left(u_{\varepsilon}\right) \leq m_{e}^{+}+\delta<2 m_{e}^{+}$. Then, when $\varepsilon$ is sufficiently small, $u_{\varepsilon}$ has a unique maximum point $P \in \partial M$.

Proof. We argue by contradiction. Suppose that $u_{\varepsilon}$ has two maximum points $P_{\varepsilon}^{1}, P_{\varepsilon}^{2} \in \partial M$. We first prove that $d_{g}\left(P_{\varepsilon}^{1}, P_{\varepsilon}^{2}\right) \rightarrow 0$.

Otherwise, we can find a sequence of vanishing positive numbers $\varepsilon_{j}$ and for each $\varepsilon_{j}$ a solution $u_{\varepsilon_{j}}$ with (at least) two maximum points $P_{\varepsilon_{j}}^{1} \rightarrow P^{1}$ and $P_{\varepsilon_{j}}^{2} \rightarrow P^{2}$ as $j \rightarrow \infty$ with $P^{1} \neq P^{2}$. 
We define $Q_{\varepsilon_{j}}^{i} \in \mathbb{R}^{n-1}$ such that

$$
P_{\varepsilon_{j}}^{i}=\exp _{P^{i}}^{\partial}\left(Q_{\varepsilon_{j}}^{i}\right) \quad i=1,2
$$

and we can define a sequence $v_{j}^{1}$ as

$$
v_{j}^{1}(z)=\left\{\begin{array}{cc}
u_{\varepsilon_{j}}\left(\psi_{P^{1}}^{\partial}\left(Q_{\varepsilon_{j}}^{1}+\varepsilon_{j} z\right)\right) & \text { for } z_{n} \geq 0 \\
u_{\varepsilon_{j}}\left(\psi_{P^{1}}^{\partial}\left(Q_{\varepsilon_{j}}^{1}+\varepsilon_{j} z^{\tau}\right)\right) & \text { for } z_{n}<0
\end{array}\right.
$$

where $z^{\tau}=\left(z_{1}, \ldots, z_{n-1},-z_{n}\right)$, and $z \in \mathbb{R}^{n}$ sufficiently small such that the Fermi coordinates $\psi_{P^{1}}^{\partial}$ are well defined. In the same way we define $v_{j}^{2}$. At this point we can proceed as in 13 and we can prove that for any bounded set $B$ eventually $v_{j}^{i} \in C^{2}(B)$ and $v_{j}^{i} \stackrel{j}{\rightarrow} U$ in $C^{2}(B)$, where $U$ is the positive, radially symmetric least energy solution of (5). Now choose $\bar{R}$ such that

$$
\int_{B(0, \bar{R})}|\nabla U|^{2}+\left(a-\omega^{2}\right) U^{2}>\frac{2 p}{p-2} \cdot \frac{m_{\infty}+2 \delta}{2} .
$$

For $\varepsilon_{j}$ sufficiently small, we have that $\varepsilon_{j} \bar{R} \leq \frac{d_{g}\left(P^{1}, P^{2}\right)}{2}$, thus

$$
\begin{aligned}
2 I_{\varepsilon_{j}}\left(u_{\varepsilon_{j}}\right) \geq 2 & \left(\frac{1}{2}-\frac{1}{p}\right)\left\|u_{\varepsilon_{j}}\right\|_{\varepsilon_{j}}^{2} \\
\geq & 2\left(\frac{1}{2}-\frac{1}{p}\right) \frac{1}{\varepsilon_{j}^{n}} \int_{I_{P^{1}}\left(\varepsilon_{j} \bar{R}\right) \cup I_{P^{2}}\left(\varepsilon_{j} \bar{R}\right)} \varepsilon^{2}\left|\nabla_{g} u_{\varepsilon_{j}}\right|^{2}+\left(a-\omega^{2}\right) u_{\varepsilon_{j}}^{2} \\
\geq & 2\left(\frac{1}{2}-\frac{1}{p}\right) \int_{B(0, \bar{R}) \cap z_{n} \geq 0}\left|\nabla v_{j}^{1}(z)\right|^{2}+\left(a-\omega^{2}\right)\left(v_{j}^{1}\right)^{2} \\
& +2\left(\frac{1}{2}-\frac{1}{p}\right) \int_{B(0, \bar{R}) \cap z_{n} \geq 0}\left|\nabla v_{j}^{2}(z)\right|^{2}+\left(a-\omega^{2}\right)\left|v_{j}^{2}\right|^{2}+o(1) \\
= & \left(\frac{1}{2}-\frac{1}{p}\right) \int_{B(0, \bar{R})}\left|\nabla v_{j}^{1}(z)\right|^{2}+\left(a-\omega^{2}\right)\left|v_{j}^{1}\right|^{2} \\
& +\left(\frac{1}{2}-\frac{1}{p}\right) \int_{B(0, \bar{R})}\left|\nabla v_{j}^{2}(z)\right|^{2}+\left(a-\omega^{2}\right)\left|v_{j}^{2}\right|^{2}+o(1) \\
\rightarrow 2 & \left(\frac{1}{2}-\frac{1}{p}\right) \int_{B(0, \bar{R})}|\nabla U|^{2}+\left(a-\omega^{2}\right) U^{2}>m_{\infty}+2 \delta
\end{aligned}
$$

and thus $I_{\varepsilon_{j}}\left(u_{\varepsilon_{j}}\right)>m_{e}^{+}+2 \delta$ that is a contradiction.

Now we have that $d_{g}\left(P_{\varepsilon}^{1}, P_{\varepsilon}^{2}\right) \rightarrow 0$. With the same technique we can prove also that

$$
\lim _{j \rightarrow \infty} \frac{1}{\varepsilon_{j}} d_{g}\left(P_{\varepsilon_{j}}^{1}, P_{\varepsilon_{j}}^{2}\right)=0
$$

To conclude the proof we have to show that (33) raises to a contradiction. In fact suppose that $d_{g}\left(P_{\varepsilon_{j}}^{1}, P_{\varepsilon_{j}}^{2}\right) \leq c \varepsilon_{j}$ for some $c>0$ and consider the sequence of functions

$$
w_{\varepsilon_{j}}=\left\{\begin{array}{cl}
u_{\varepsilon_{j}}\left(\psi_{P^{1}}^{\partial}\left(Q_{\varepsilon_{j}}^{1}+\varepsilon_{j} z\right)\right) & \text { for } z_{n} \geq 0 \\
u_{\varepsilon_{j}}\left(\psi_{P^{1}}^{\partial}\left(Q_{\varepsilon_{j}}^{1}+\varepsilon_{j} z^{\tau}\right)\right) & \text { for } z_{n}<0
\end{array} \text { with }|z| \leq c .\right.
$$


For any $j, w_{\varepsilon_{j}}$ has two maximum points in $B(0, c)$. Moreover, we can argue, as in the previous steps, that $w_{\varepsilon_{j}} \rightarrow U$ in $C^{2}(B(0, c))$ and this is a contradiction.

Lemma 18. Write $u_{\varepsilon}=Z_{\varepsilon, P_{\varepsilon}}+\Psi_{\varepsilon}$ where $Z_{\varepsilon, P_{\varepsilon}}$ is defined in (6) and $P_{\varepsilon} \in \partial M$ is the unique maximum point. It holds that $\left\|\Psi_{\varepsilon}\right\|_{L^{\infty}(M)} \rightarrow 0$.

Proof. By the $C^{2}$ convergence proved in Lemma 17 we have that, given $\rho>0$, and defined $w_{\varepsilon}$ as in (34), we get, as before,

$$
2\left\|u_{\varepsilon}-Z_{\varepsilon, P_{\varepsilon}}\right\|_{C^{0}\left(I_{P_{\varepsilon}}(\varepsilon \rho)\right)}=\left\|w_{\varepsilon}(z)-U(z)\right\|_{C^{0}(B(0, \rho))}+o(1) \rightarrow 0
$$

as $\varepsilon \rightarrow 0$. Moreover, since $u_{\varepsilon}$ has a unique maximum point by Lemma 17, we have that, for any $\rho>0$,

$$
\max _{x \in M \backslash I_{P_{\varepsilon}}(\varepsilon \rho)} u_{\varepsilon}(x)=\max _{x \in \partial I_{P_{\varepsilon}}(\varepsilon \rho)} u_{\varepsilon}(x)=\max _{|z|=\rho} U(z)+\sigma(\varepsilon) \leq c e^{-\alpha \rho}+\sigma_{1}(\varepsilon)
$$

for some constant $c, \alpha>0$ and for some $\sigma_{1}(\varepsilon) \rightarrow 0$ for $\varepsilon \rightarrow 0$. This proves the claim.

\section{Proof of technical Results}

Here we collect some technical result which has been used in the proof of the main result.

Proof of Lemma 6. If $u \in \mathcal{N}_{\varepsilon}$, , by (15), we have

$$
\begin{aligned}
0=N_{\varepsilon}(u) & =\|u\|_{\varepsilon}^{2}-\left|u^{+}\right|_{\varepsilon, p}^{p}+\frac{q \omega^{2}}{\varepsilon^{n}} \int_{M}(2-q \psi(u)) \psi(u) u^{2} d \mu_{g} \\
& =\|u\|_{\varepsilon}^{2}-\left|u^{+}\right|_{\varepsilon, p}^{p}+\frac{q \omega^{2}}{2 \varepsilon^{n}} \int_{M}\left(2 \psi(u)+\psi^{\prime}(u)[u]\right) u^{2} d \mu_{g} .
\end{aligned}
$$

The functional $N_{\varepsilon}$ is of class $C^{2}$ for $2<p<2^{*}$ because $\psi$ is of class $C^{2}$. Also, for $4 \leq p<2^{*}$ we have $N_{\varepsilon}^{\prime}(u)[u]<0$ for all $u \in \mathcal{N}_{\varepsilon}$. In fact by (35) we have

$$
\begin{aligned}
N_{\varepsilon}^{\prime}(u)[u]= & 2\|u\|_{\varepsilon}^{2}-p\left|u^{+}\right|_{\varepsilon, p}^{p}+\frac{q \omega^{2}}{\varepsilon^{n}} \int_{M}(2-q \psi(u)) \psi^{\prime}(u)[u] u^{2} d \mu_{g} \\
& +\frac{2 q \omega^{2}}{\varepsilon^{n}} \int_{M}(2-q \psi(u)) \psi(u) u^{2} d \mu_{g}-\frac{q^{2} \omega^{2}}{\varepsilon^{n}} \int_{M} \psi^{\prime}(u)[u] \psi(u) u^{2} d \mu_{g}= \\
= & (2-p)\|u\|_{\varepsilon}^{2}+\frac{q \omega^{2}}{\varepsilon^{n}} \int_{M}[4-p-2 q \psi(u)] \psi(u) u^{2} d \mu_{g} \\
& +\frac{q \omega^{2}}{\varepsilon^{n}} \int_{M}\left[2-\frac{p}{2}-2 q \psi(u)\right] \psi^{\prime}(u)[u] u^{2} d \mu_{g}<0 \text { for } p \geq 4,
\end{aligned}
$$

thus $\mathcal{N}_{\varepsilon}$ is a $C^{2}$ manifold.

Now, assume by contradiction that there exists a sequence $\left\{u_{k}\right\}_{k} \in \mathcal{N}_{\varepsilon}$ with $\left\|u_{k}\right\|_{\varepsilon} \rightarrow 0$ while $k \rightarrow+\infty$. Thus, using that $N_{\varepsilon}(u)=0$ and that $0 \leq \psi\left(u_{k}\right) \leq 1 / q$ we have

$$
\left\|u_{k}\right\|_{\varepsilon}^{2} \leq\left\|u_{k}\right\|_{\varepsilon}^{2}+\frac{q \omega^{2}}{\varepsilon^{n}} \int_{M}\left[2-q \psi\left(u_{k}\right)\right] u_{k}^{2} \psi\left(u_{k}\right) d \mu_{g}=\left|u_{k}^{+}\right|_{p, \varepsilon}^{p} \leq C\left\|u_{k}\right\|_{\varepsilon}^{p},
$$

so $1 \leq C\left\|u_{k}\right\|_{\varepsilon}^{p-2} \rightarrow 0$ that gives us a contradiction, so claim 1 is proved.

To prove claim 2, first, we show that if $\left\{u_{k}\right\}_{k} \in \mathcal{N}_{\varepsilon}$ is a Palais-Smale sequence for the functional $I_{\varepsilon}$ constrained on $\mathcal{N}_{\varepsilon}$, then $\left\{u_{k}\right\}_{k}$ is a is a Palais-Smale sequence for the free functional $I_{\varepsilon}$ on $H_{\varepsilon}$ 
Indeed, let $\left\{u_{k}\right\}_{k} \in \mathcal{N}_{\varepsilon}$ such that

$$
\begin{gathered}
I_{\varepsilon}\left(u_{k}\right) \rightarrow c \\
\left|I_{\varepsilon}^{\prime}\left(u_{k}\right)[\varphi]-\lambda_{k} N^{\prime}\left(u_{k}\right)[\varphi]\right| \leq \sigma_{k}\|\varphi\|_{\varepsilon} \quad \text { with } \sigma_{k} \rightarrow 0
\end{gathered}
$$

In particular $I_{\varepsilon}^{\prime}\left(u_{k}\right)\left[\frac{u_{k}}{\left\|u_{k}\right\|_{\varepsilon}}\right]-\lambda_{k} N^{\prime}\left(u_{k}\right)\left[\frac{u_{k}}{\left\|u_{k}\right\|_{\varepsilon}}\right] \rightarrow 0$. Thus, since $u_{k} \in \mathcal{N}_{\varepsilon}$,

$$
\lambda_{k} N^{\prime}\left(u_{k}\right)\left[\frac{u_{k}}{\left\|u_{k}\right\|_{\varepsilon}}\right] \rightarrow 0 .
$$

By (36), if inf $\left|\lambda_{k}\right| \neq 0$, we have that $\left\|u_{k}\right\|_{\varepsilon} \rightarrow 0$ that contradicts Lemma [6] Thus $\lambda_{k} \rightarrow 0$. Moreover, since

$$
I_{\varepsilon}\left(u_{k}\right)=\left(\frac{1}{2}-\frac{1}{p}\right)\left\|u_{k}\right\|_{\varepsilon}^{2}+\left(\frac{1}{2}-\frac{2}{p}\right) \frac{\omega^{2} q}{\varepsilon^{3}} \int_{M} u_{k}^{2} \psi_{k} d \mu_{g}+\frac{\omega^{2} q^{2}}{\varepsilon^{n} p} \int_{M} u_{k}^{2} \psi_{k}^{2} d \mu_{g} \rightarrow c,
$$

we have that $\left\|u_{n}\right\|_{\varepsilon}$ is bounded. By Remark 20 we have that $\left|N^{\prime}\left(u_{n}\right)[\varphi]\right| \leq c\|\varphi\|_{\varepsilon}$. Thus $\left\{u_{k}\right\}_{k}$ is a PS sequence for the free functional $I_{\varepsilon}$.

To conclude the proof of claim 2, we prove that $I_{\varepsilon}$ safisfies the PS condition on the whole space $H_{\varepsilon}$. Let $\left\{u_{k}\right\}_{k} \in H_{\varepsilon}$ such that

$$
I_{\varepsilon}\left(u_{k}\right) \rightarrow c \quad\left|I_{\varepsilon}^{\prime}\left(u_{k}\right)[\varphi]\right| \leq \sigma_{k}\|\varphi\|_{\varepsilon} \text { where } \sigma_{k} \rightarrow 0
$$

We have that $\left\|u_{k}\right\|_{\varepsilon}$ is bounded. Indeed, by contradiction, suppose $\left\|u_{n}\right\|_{\varepsilon} \rightarrow \infty$. Then, by PS hypothesis

$$
\begin{aligned}
& \frac{p I_{\varepsilon}\left(u_{k}\right)-I_{\varepsilon}^{\prime}\left(u_{k}\right)\left[u_{k}\right]}{\left\|u_{k}\right\|_{\varepsilon}}= \\
&\left(\frac{p}{2}-1\right)\left\|u_{k}\right\|_{\varepsilon}+\frac{q \omega^{2}}{\varepsilon^{n}} \int_{M}\left[\frac{p}{2}-2+q \psi\left(u_{k}\right)\right] \frac{u_{k}^{2} \psi\left(u_{k}\right)}{\left\|u_{k}\right\|_{\varepsilon}} d \mu_{g} \rightarrow 0
\end{aligned}
$$

Since $p \geq 4$ and $\psi\left(u_{n}\right) \geq 0$ this leads to a contradiction. At this point, up to subsequence $u_{k} \rightarrow u$ in $H_{\varepsilon}$, then by Lemma 19 we have, up to subsequence, $\psi\left(u_{k}\right):=$ $\psi_{k} \rightarrow \bar{\psi}=\psi(u)$.

We have that

$$
u_{k}-i_{\varepsilon}^{*}\left[\left(u_{k}^{+}\right)^{p-1}\right]-\omega^{2} q i_{\varepsilon}^{*}\left[\left(q \psi_{k}^{2}-2 \psi_{k}\right) u_{k}\right] \rightarrow 0
$$

where the operator $i_{\varepsilon}^{*}: L_{g}^{p^{\prime}},|\cdot|_{\varepsilon, p^{\prime}} \rightarrow H_{\varepsilon}$ is the adjoint operator of the immersion operator $i_{\varepsilon}: H_{\varepsilon} \rightarrow L_{g}^{p},|\cdot|_{\varepsilon, p}$. Since $u_{k} \rightarrow u$ in $L^{p^{\prime}}$, to get $H_{g}^{1}$ strong convergence of $\left\{u_{k}\right\}_{k}$ it is sufficient to show that $\left(q \psi_{k}^{2}-2 \psi_{k}\right) u_{n} \rightarrow\left(q \bar{\psi}^{2}-2 \bar{\psi}\right) u$ in $L_{g}^{p^{\prime}}$. We have

$$
\left|\psi_{k} u_{k}-\bar{\psi} u\right|_{p^{\prime}, g} \leq\left|\left(\psi_{k}-\bar{\psi}\right) u\right|_{p^{\prime}, g}+\left|\psi_{k}\left(u_{k}-u\right)\right|_{p^{\prime}, g} \text {. }
$$

and

$$
\left|\psi_{k}^{2} u_{k}-\bar{\psi}^{2} u\right|_{p^{\prime}, g} \leq\left|\left(\psi_{k}^{2}-\bar{\psi}^{2}\right) u\right|_{p^{\prime}, g}+\left|\psi_{k}^{2}\left(u_{k}-u\right)\right|_{p^{\prime}, g} .
$$

For the first term of (37) we have, by Holder inequality

$$
\int_{M}\left|\psi_{k}-\bar{\psi}\right|^{\frac{p}{p-1}}|u|^{\frac{p}{p-1}} \leq\left(\int_{M}\left|\psi_{k}-\bar{\psi}\right|^{p}\right)^{\frac{1}{p-1}}\left(\int_{M}|u|^{\frac{p}{p-2}}\right)^{\frac{p-2}{p-1}} \rightarrow 0,
$$

and for the other terms we proceed in the same way.

To prove claim 3 , define, for $t>0$

$$
H(t)=I_{\varepsilon}(t u)=\frac{1}{2} t^{2}\|u\|_{\varepsilon}^{2}+\frac{q \omega^{2}}{2 \varepsilon^{n}} t^{2} \int_{M} \psi(t u) u^{2} d \mu_{g}-\frac{t^{p}}{p} .
$$


Thus, by (15)

$$
\begin{aligned}
H^{\prime}(t)= & t\left(\|u\|_{\varepsilon}^{2}+\frac{q \omega^{2}}{2 \varepsilon^{n}} \int_{M}[2-q \psi(t u)] \psi(t u) u^{2} d \mu_{g}-t^{p-2}\right) \\
= & t\left(\|u\|_{\varepsilon}^{2}+\frac{q \omega^{2}}{\varepsilon^{n}} \int_{M} \psi(t u) u^{2} d \mu_{g}+\frac{q \omega^{2}}{2 \varepsilon^{n}} t \int_{M} \psi^{\prime}(t u)[u] u^{2} d \mu_{g}-t^{p-2}\right) \\
H^{\prime \prime}(t)= & \|u\|_{\varepsilon}^{2}+\frac{q \omega^{2}}{2 \varepsilon^{n}} \int_{M}[2-q \psi(t u)] \psi(t u) u^{2} d \mu_{g} \\
& +\frac{q \omega^{2}}{\varepsilon^{n}} t \int_{M}[1-q \psi(t u)] \psi^{\prime}(t u)[u] u^{2} d \mu_{g}-(p-1) t^{p-2}
\end{aligned}
$$

By (39) there exists $t_{\varepsilon}>0$ such that $H^{\prime}\left(t_{\varepsilon}\right)=0$, because, for small $t, H^{\prime}(t)>0$ and, since $p \geq 4$, it holds $H^{\prime}(t)<0$ for $t$ large. Moreover,

$$
t_{\varepsilon}^{p-2}=\|u\|_{\varepsilon}^{2}+\frac{q \omega^{2}}{\varepsilon^{n}} \int_{M} \psi\left(t_{\varepsilon} u\right) u^{2} d \mu_{g}+\frac{q \omega^{2}}{2 \varepsilon^{n}} t_{\varepsilon} \int_{M} \psi^{\prime}\left(t_{\varepsilon} u\right)[u] u^{2} d \mu_{g}
$$

then, by Lemma 4

$$
\begin{aligned}
H^{\prime \prime}\left(t_{\varepsilon}\right)= & (2-p)\|u\|_{\varepsilon}^{2}+\frac{q \omega^{2}}{\varepsilon^{n}} \int_{M}\left[2-p-\frac{q}{2} \psi\left(t_{\varepsilon} u\right)\right] \psi\left(t_{\varepsilon} u\right) u^{2} d \mu_{g} \\
& +\frac{q \omega^{2}}{2 \varepsilon^{n}} \int_{M}\left[3-p-2 q \psi\left(t_{\varepsilon} u\right)\right] \psi^{\prime}\left(t_{\varepsilon} u\right)\left[t_{\varepsilon} u\right] u^{2} d \mu_{g}<0
\end{aligned}
$$

so $t_{\varepsilon}$ is unique. The continuity of $t_{\varepsilon}$ is standard.

We now prove the last claim. We have

$$
\begin{aligned}
t_{\varepsilon}^{p-2}\left|Z_{\varepsilon, \xi}\right|_{\varepsilon, p}^{p}=\left\|Z_{\varepsilon, \xi}\right\|_{\varepsilon}^{2}+\frac{q \omega^{2}}{\varepsilon^{n}} \int_{M} \psi\left(t_{\varepsilon} Z_{\varepsilon, \xi}\right) & Z_{\varepsilon, \xi}^{2} d \mu_{g} \\
& -\frac{q^{2} \omega^{2}}{2 \varepsilon^{n}} \int_{M} \psi^{2}\left(t_{\varepsilon} Z_{\varepsilon, \xi}\right) Z_{\varepsilon, \xi}^{2} d \mu_{g}
\end{aligned}
$$

where $t_{\varepsilon}=t_{\varepsilon}\left(Z_{\varepsilon, q}\right)$. It holds

$$
\begin{aligned}
& \lim _{\varepsilon \rightarrow 0} \frac{1}{\varepsilon^{n} t_{\varepsilon}^{2}} \int_{M} \psi\left(t_{\varepsilon} Z_{\varepsilon, \xi}\right) Z_{\varepsilon, \xi}^{2} d \mu_{g}=0 \\
& \lim _{\varepsilon \rightarrow 0} \frac{1}{\varepsilon^{n} t_{\varepsilon}^{4}} \int_{M} \psi^{2}\left(t_{\varepsilon} Z_{\varepsilon, \xi}\right) Z_{\varepsilon, \xi}^{2} d \mu_{g}=0
\end{aligned}
$$

In fact, set $\psi\left(t_{\varepsilon} Z_{\varepsilon, \xi}\right):=\psi_{\varepsilon}$. We have, by Remark 21 and by definition of $\psi_{\varepsilon}$,

$$
\begin{aligned}
\left\|\psi_{\varepsilon}\right\|_{H}^{2} & \leq\left\|\psi_{\varepsilon}\right\|_{H}^{2}+q^{2} \int_{M} \psi_{\varepsilon}^{2} t_{\varepsilon}^{2} Z_{\varepsilon, \xi}^{2} d \mu_{g}=t_{\varepsilon}^{2} q \int_{M} Z_{\varepsilon, \xi}^{2} \psi_{\varepsilon} d \mu_{g} \leq \\
& \leq c t_{\varepsilon}^{2}\left|\psi_{\varepsilon}\right|_{6, g}\left(\int_{M} Z_{\varepsilon, \xi}^{12 / 5} d \mu_{g}\right)^{5 / 6} \leq c t_{\varepsilon}^{2}\left\|\psi_{\varepsilon}\right\|_{H_{g}^{1}} \varepsilon^{\frac{5 n}{6}} .
\end{aligned}
$$

Moreover

$$
\frac{1}{\varepsilon^{n}} \int_{M} \psi_{\varepsilon} Z_{\varepsilon, \xi}^{2} d \mu_{g} \leq \frac{1}{\varepsilon^{n}}\left\|\psi_{\varepsilon}\right\|_{H_{g}^{1}}\left(\int_{M} Z_{\varepsilon, \xi}^{12 / 5} d \mu_{g}\right)^{5 / 6} \leq c t_{\varepsilon}^{2} \frac{1}{\varepsilon^{n}} \varepsilon^{\frac{10 n}{6}}=c t_{\varepsilon}^{2} \varepsilon^{\frac{2 n}{3}}
$$

and

$$
\frac{1}{\varepsilon^{n}} \int_{M} \psi_{\varepsilon}^{2} Z_{\varepsilon, \xi}^{2} d \mu_{g} \leq \frac{1}{\varepsilon^{n}}\left(\int \psi_{\varepsilon}^{6} d \mu_{g}\right)^{1 / 3}\left(\int_{M} Z_{\varepsilon, \xi}^{3} d \mu_{g}\right)^{2 / 3} \leq \frac{1}{\varepsilon^{n}}\left\|\psi_{\varepsilon}\right\|_{H_{g}^{1}}^{2} \varepsilon^{\frac{2 n}{3}} \leq t_{\varepsilon}^{4} \varepsilon^{\frac{4 n}{3}} .
$$


This proves (42) and (43). For any sequence $\varepsilon_{k} \rightarrow 0$, by (41), (42) and (43) and by Remark 21 we have that $t_{\varepsilon_{k}}$ is bounded. Then, up to subsequences $t_{\varepsilon_{k}} \rightarrow \bar{t}$. By (41) and Remark 21] we have $\bar{t}^{p-2}|V|_{p}^{p}=\int_{\mathbb{R}_{+}^{n}}|\nabla V|^{2}+\left(a-\omega^{2}\right) V^{2} d x$. By (44) we complete the proof.

Lemma 19. Let $u_{k} \rightarrow u$ in $H_{g}^{1}(M)$. Then, up to subsequence, $\psi\left(u_{k}\right) \rightarrow \psi(u)$ in $H_{g}^{1}(M)$.

Proof. We set $\psi_{k}:=\psi\left(u_{k}\right)$. By (7), it holds

$$
\left\|\psi_{k}\right\|_{H_{g}^{1}}^{2} \leq\left\|\psi_{k}\right\|_{H_{g}^{1}}^{2}+\int_{M} q^{2} u_{k}^{2} \psi_{k}^{2} d \mu_{g}=q \int_{M} u_{k}^{2} \psi_{k} d \mu_{g} \leq c\left\|u_{k}\right\|_{L_{g}^{4}}^{2}\left\|\psi_{k}\right\|_{H_{g}^{1}}
$$

then $\left\|\psi_{k}\right\|_{H_{g}^{1}} \leq c\left\|u_{k}\right\|_{L_{g}^{4}}^{2}$, thus $\left\|\psi_{k}\right\|_{H_{g}^{1}}$ is bounded and, up to subsequence, $\psi_{k} \rightarrow \bar{\psi}$ in $H_{g}^{1}(M)$. We recall that $\psi_{k}$ solves (7), thus passing to the limit we have that $\bar{\psi}$ also solves (77). Since (7) admits a unique solution, we get $\bar{\psi}=\psi(u)$. If $\psi\left(u_{k}\right)$ solves (8) the proof follows in the same way if we use on $H_{0, g}^{1}$ the equivalent norm $\|u\|_{H_{0, g}^{1}}=\|\nabla u\|_{L_{g}^{2}}$.

Remark 20. We have that $\left\|V_{u}(h)\right\|_{H} \leq c|h|_{3, g}|u|_{3, g}$. In fact, by Lemma 4

$$
\begin{aligned}
\left\|V_{u}(h)\right\|_{H}^{2} & \leq\left\|V_{u}(h)\right\|_{H}^{2}+\int_{M} q^{2} u^{2} V_{u}^{2}(h) d \mu_{g} \leq \\
& \leq \int_{M} 2 q u(1-q \psi(u)) h V_{u}(h) d \mu_{g} \leq c\left\|V_{u}(h)\right\|_{H}|h|_{3, g}|u|_{3, g} .
\end{aligned}
$$

Remark 21. the following limits hold uniformly with respect to $q \in \partial M$.

$$
\begin{aligned}
\lim _{\varepsilon \rightarrow 0}\left\|Z_{\varepsilon, \xi}\right\|_{2, \varepsilon}^{2} & =\int_{\mathbb{R}_{+}^{n}} V^{2}(y) d y \\
\lim _{\varepsilon \rightarrow 0}\left\|Z_{\varepsilon, \xi}\right\|_{p, \varepsilon}^{p} & =\int_{\mathbb{R}_{+}^{n}} V^{p}(y) d y \\
\lim _{\varepsilon \rightarrow 0} \varepsilon^{2}\left\|\nabla Z_{\varepsilon, \xi}\right\|_{2, \varepsilon}^{2} & =\int_{\mathbb{R}_{+}^{n}}|\nabla V|^{2}(y) d y
\end{aligned}
$$

\section{REFERENCES}

[1] Antonio Azzollini and Alessio Pomponio, Ground state solutions for the nonlinear kleingordon-maxwell equations, Topol. Methods Nonlinear Anal. 35 (2010), 33-42.

[2] Vieri Benci and Donato Fortunato, Solitary waves of the nonlinear Klein-Gordon equation coupled with the Maxwell equations, Rev. Math. Phys. 14 (2002), no. 4, 409-420. MR 1901222 (2003f:35079)

[3] Daniele Cassani, Existence and non-existence of solitary waves for the critical Klein-Gordon equation coupled with Maxwell's equations, Nonlinear Anal. 58 (2004), no. 7-8, 733-747. MR 2085333 (2005f:35297)

[4] Pascal Cherrier, Problèmes de Neumann non linéaires sur les variétés riemanniennes, J. Funct. Anal. 57 (1984), no. 2, 154-206. MR 749522 (86c:58154)

[5] Tea D'Aprile and Juncheng Wei, Layered solutions for a semilinear elliptic system in a ball, J. Differential Equations 226 (2006), 269-294.

[6] Tea D'Aprile and Juncheng Wei, Clustered solutions around harmonic centers to a coupled elliptic system, Ann. Inst. H. Poincaré Anal. Non Linéaire 226 (2007), 605-628.

[7] Teresa D'Aprile and Dimitri Mugnai, Solitary waves for nonlinear Klein-Gordon-Maxwell and Schrödinger-Maxwell equations, Proc. Roy. Soc. Edinburgh Sect. A 134 (2004), no. 5, 893-906. MR 2099569 (2005h:35319) 
[8] P. d'Avenia, L. Pisani, and G. Siciliano, Dirichlet and Neumann problems for Klein-GordonMaxwell systems, Nonlinear Anal. 71 (2009), no. 12, e1985-e1995. MR 2671970 (2011e:35317)

[9] Pietro d'Avenia and Lorenzo Pisani, Nonlinear Klein-Gordon equations coupled with BornInfeld type equations, Electron. J. Differential Equations (2002), No. 26, 13. MR 1884995 (2003e:78006)

[10] Pietro d'Avenia, Lorenzo Pisani, and Gaetano Siciliano, Klein-Gordon-Maxwell systems in a bounded domain, Discrete Contin. Dyn. Syst. 26 (2010), no. 1, 135-149. MR 2552782 (2010j:35528)

[11] Olivier Druet and Emmanuel Hebey, Existence and a priori bounds for electrostatic KleinGordon-Maxwell systems in fully inhomogeneous spaces, Commun. Contemp. Math. 12 (2010), no. 5, 831-869. MR 2733200 (2012j:58018)

[12] Marco Ghimenti and Anna M. Micheletti, Positive solutions of singularly perturbed nonlinear elliptic problem on Riemannian manifolds with boundary, Topol. Methods Nonlinear Anal. 35 (2010), no. 2, 319-337. MR 2676820 (2011i:58028)

[13] Marco Ghimenti and Anna Maria Micheletti, Number and profile of low energy solutions for singularly perturbed Klein-Gordon-Maxwell systems on a Riemannian manifold, J. Differential Equations 256 (2014), no. 7, 2502-2525. MR 3160452

[14] Emmanuel Hebey and Trong Tuong Truong, Static Klein-Gordon-Maxwell-Proca systems in 4-dimensional closed manifolds, J. Reine Angew. Math. 667 (2012), 221-248. MR 2929678

[15] Emmanuel Hebey and Juncheng Wei, Resonant states for the static Klein-Gordon-MaxwellProca system, Math. Res. Lett. 19 (2012), no. 4, 953-967. MR 3008428

[16] Dimitri Mugnai, Coupled klein-gordon and born-infeld-type equations: Looking for solitary waves, Proc. R. Soc. Lond. Ser. A Math. Phys. Eng. Sci. 460 (2004), 1519-1527.

(Marco Ghimenti) Dipartimento di Matematica, Università di Pisa, via F. Buonarroti $1 / \mathrm{C}, 56127$ Pisa, ItALy

E-mail address: marco.ghimenti@dma.unipi.it.

(Anna Maria Micheletti) Dipartimento di Matematica, Università di Pisa, via F. BuonarRoti $1 / \mathrm{C}, 56127$ Pisa, ItALy

E-mail address: a.micheletti@dma.unipi.it. 